\title{
Fluctuations and correlations in hexagonal optical patterns
}

\author{
Damià Gomila and Pere Colet \\ Institut Mediterrani d'Estudis Avançats, IMEDEA (CSIC-UIB), Campus Universitat Illes Balears, E-07071 Palma de Mallorca, Spain
}

(Received 3 July 2002; published 30 October 2002)

\begin{abstract}
We analyze the influence of noise in transverse hexagonal patterns in nonlinear Kerr cavities. The near-field fluctuations are determined by the neutrally stable Goldstone modes associated to translational invariance and by the weakly damped soft modes. However, these modes do not contribute to the far-field intensity fluctuations that are dominated by damped perturbations with the same wave vectors than the pattern. We find strong correlations between the intensity fluctuations of any arbitrary pair of wave vectors of the pattern. Correlation between pairs forming $120^{\circ}$ is larger than between pairs forming $180^{\circ}$, contrary to what a naive interpretation of emission in terms of twin photons would suggest.
\end{abstract}

DOI: 10.1103/PhysRevE.66.046223

PACS number(s): 05.40.-a, 47.54.+r, 42.65.Sf

\section{INTRODUCTION}

The properties of the fluctuations and correlations in spatially extended patterns out of thermodynamical equilibrium were studied long ago in the context of hydrodynamic systems $[1,2]$. More recently, there has been a new surge of interest in the field of nonlinear and quantum optics since Lugiato and Castelli pointed out the existence of a purely quantum phenomenon in a spatial stationary dissipative structure [3], the reduction of fluctuations below quantum limits in the difference between the intensities of the two Fourier modes of a stripe pattern. Since then, the properties of the fluctuations and correlations of stripe patterns in different nonlinear optical models have been widely studied [4-7]. Stripe patterns in these systems appear as supercritical transitions, and close to the threshold for pattern formation, the harmonics of the fundamental wave vectors can be neglected. Therefore considering the homogeneous mode and the two modes of the stripes for each field is enough and simplifies the problem, allowing for an analytic treatment. The problem considering the whole infinite set of transverse modes in optical stripe patterns has been addressed numerically in Ref. [7]. At difference with the few-mode approximation, the continuous problem makes evident the role of the so-called soft modes in the fluctuations of the near field. However, a very good agreement between the few-mode approximation and the continuous treatment is still found for the correlations of the fluctuations of the far-field mode intensities close to threshold.

Due to the additional complexity, fluctuations and correlations in hexagonal optical patterns have been much less studied $[8,9]$. Furthermore, these two papers use an approximation in few modes to describe the pattern. However hexagonal patterns generally appear subcritically, with finite amplitude. Therefore, harmonics are not negligible even at threshold. Strictly speaking, an approximation in which only the homogeneous mode plus the six modes of the hexagons are considered is not fully justified.

In this paper, we will treat the case of a subcritical hexagonal pattern using a continuous model, i.e., avoiding any restriction to a reduced number of spatial modes. In particular, we consider an optical cavity filled with a nonlinear isotropic Kerr medium. This situation is described by the
Lugiato-Lefever model [10] in which a Turing instability was described for the self-focusing case. This patternforming instability leads to a hexagonal pattern in the twodimensional transverse plane. We find that the fluctuation of the near field are dominated by the two Goldstone modes associated to the translational invariance in the $x$ and $y$ directions, and by the soft modes arbitrarily close to them in infinitely large systems. We also show that the modes that dominate the near-field fluctuations do not contribute at all to the far-field intensity fluctuations. We identify the modes responsible for the far-field intensity correlations, and we find (a) strong correlations between arbitrary pairs of wave vectors of the pattern, but stronger between those forming a $120^{\circ}$ angle; (b) anticorrelation between the zero wave vector of the spectrum of fluctuations and any wave vector of the pattern. While the anti-correlation of the homogeneous mode with the off-axis wave vectors can be understood in terms of energy conservations, the common microscopic interpretation of the far-field intensity correlations in terms of emission of twin photons would naively suggest that the strongest correlation is between the wave vectors forming a $180^{\circ}$ angle. In fact, the total transverse momentum conservation always involves at least four modes simultaneously and give some hints about how the correlations should be, but does not identify the pairs with stronger correlations. Our results here are obtained within a semiclassical approach in which specific features of quantum statistics are neglected.

The paper is organized as follows: In Sec. II we describe the model we are considering. In Sec. III we linearize around the hexagonal pattern and describe the linear response of the system to noise perturbations. In Sec. IV we discuss in detail the field fluctuations, and in Sec. V we describe the correlations of the field Fourier components. Finally, in Sec. VI we give some concluding remarks.

\section{DESCRIPTION OF THE MODEL}

The dynamics of the electric field inside an optical cavity with a self-focusing Kerr medium can be described, in the mean-field approximation, by a equation for the scaled slowly varying amplitude of the field $E(\vec{x})[10,11]$,

$$
\partial_{t} E=-(1+i \theta) E+i \nabla^{2} E+E_{0}+i 2|E|^{2} E+\xi(\vec{x}, t),
$$


where $E_{0}$ is the input field, $\theta$ is the cavity detuning, $\nabla^{2}$ is the transverse Laplacian, and $\xi(\vec{x}, t)$ is a complex Gaussian white noise with zero mean and correlations,

$$
\begin{gathered}
\left\langle\xi(\vec{x}, t) \xi^{*}\left(\vec{x}^{\prime}, t^{\prime}\right)\right\rangle=\epsilon \delta\left(\vec{x}-\vec{x}^{\prime}\right) \delta\left(t-t^{\prime}\right), \\
\left\langle\xi(\vec{x}, t) \xi\left(\vec{x}^{\prime}, t^{\prime}\right)\right\rangle=0 .
\end{gathered}
$$

In the absence of noise, Eq. (1) has a homogeneous stationary solution $E_{s}$, given by

$$
E_{0}=E_{s}\left[1-i\left(2 I_{s}-\theta\right)\right],
$$

where $I_{s}=\left|E_{s}\right|^{2}$. It is well known that the homogeneous solution (3) shows bistability for $\theta>\sqrt{3}$. We will restrict ourselves to the nonbistable regime $\theta<\sqrt{3}$.

A linear stability analysis of the homogeneous solution with respect to spatially periodic perturbations yields to the dispersion relation,

$$
\lambda(\vec{k})=-1 \pm \sqrt{-\left(\theta+k^{2}-6 I_{s}\right)\left(\theta+k^{2}-2 I_{s}\right)},
$$

where $\lambda(\vec{k})$ is the linear growth rate of a perturbation with wave vector $\vec{k}$ and $k=|\vec{k}|$. The instability threshold is located at $I_{s}^{c}=1 / 2$ and the critical wave number is $k_{c}=\sqrt{-\theta+2}$. For pump intensities above threshold, the maximum linear growth rate is for wave vectors with modulus

$$
k_{u}=\sqrt{-\theta+4 I_{s}} .
$$

At threshold $k_{u}=k_{c}$. Starting from the homogeneous solution and changing the pump intensity to a value above, but close to, the threshold, a hexagonal pattern with a wave number $k$ close to $k_{u}$ arises [which as follows from Eqs. (5) and (3) depends on the pump intensity]. The transition is subcritical, and the hexagonal pattern, once it is formed, is stable for values of the pump intensity within a quite large range that includes values below threshold. Typically, the hexagons appear oscillating just above threshold. The amplitude of the oscillations decreases on decreasing the pump intensity until they become, in all cases, stationary. We are interested here in the properties of the fluctuations and correlations of the stationary hexagonal patterns. The oscillatory behavior of the hexagons is investigated in Ref. [12]. Due to its subcriticality, the hexagonal pattern has always a finite amplitude, even at threshold. The harmonics of the six fundamental wave vectors also have a significant amplitude, so that they have to be included in the calculations to obtain realistic quantitative results. This is particularly relevant here due to the selffocusing effect, which leads to a pattern with high peaks and a strongly anharmonic far field (see Fig. 1).

\section{LINEARIZATION AROUND THE HEXAGONAL PATTERN}

The stationary hexagonal pattern can be written in the form
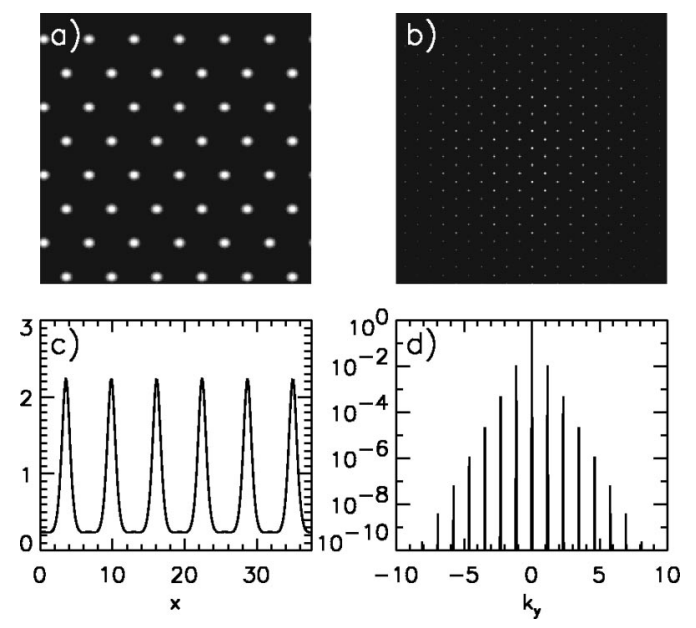

FIG. 1. (a) Near-field intensity $|E(x, y)|^{2}$ and (b) power spectrum (far field) $|E(\vec{k})|^{2}$ of a stationary hexagonal solution. Note the presence of high order harmonics; in the far field (c) shows a cross section along the $x$ axis of the intensity pattern and (d) a cross section along the $k_{y}$ axis of the power spectrum.

$$
E_{h}(\vec{x})=\sum_{n=0}^{N} a_{n} e^{i \vec{k}_{n}^{0}\left(\vec{x}-\vec{x}_{0}\right)}
$$

where $a_{n}$ are complex coefficients, $\vec{x}_{0}$ determines the global position of the pattern (we take $\vec{x}_{0}=0$ in the following), $\vec{k}_{0}^{0}$ is the homogeneous mode, and $\vec{k}_{n}^{0}$ for $n=1, \ldots, N$ are the offaxis wave vectors of the hexagonal pattern. Here we take $N=90$, which corresponds to considering up to the fifthorder harmonics in the far field. The six fundamental harmonics have modulus $k_{u}$. For simplicity we first consider the case without noise. Linearizing Eq. (1) around the stationary solution (6), we obtain the following equation for the fluctuations $\delta E(\vec{x}, t)=E(\vec{x}, t)-E_{h}(\vec{x})$,

$$
\partial_{t} \delta E=-(1+i \theta) \delta E+i \nabla^{2} \delta E+i 2\left[2\left|E_{h}\right|^{2} \delta E+E_{h} E_{h} \delta E^{*}\right] .
$$

As Eq. (7) is a linear differential equation with periodic coefficients, a general bounded solution can be found under a Floquet form [13]:

$$
\delta E(\vec{x}, t)=\int e^{i \vec{q} \vec{x}} A(\vec{q}, \vec{x}, t) d \vec{q},
$$

where $A(\vec{q}, \vec{x}, t)$ are functions with the same spatial periodicity than the stationary pattern $E_{h}$, and therefore can be written as

$$
A(\vec{q}, \vec{x}, t)=\sum_{n=0}^{N} \delta a_{n}(\vec{q}, t) e^{i \vec{k} n_{n}^{2} \vec{x}}
$$

From Eqs. (7), (8), and (9) we obtain for each perturbation wave vector $\vec{q}$ a set of linear differential equations for the time evolution of the Fourier coefficients $\delta a_{n}(\vec{q}, t)$. 


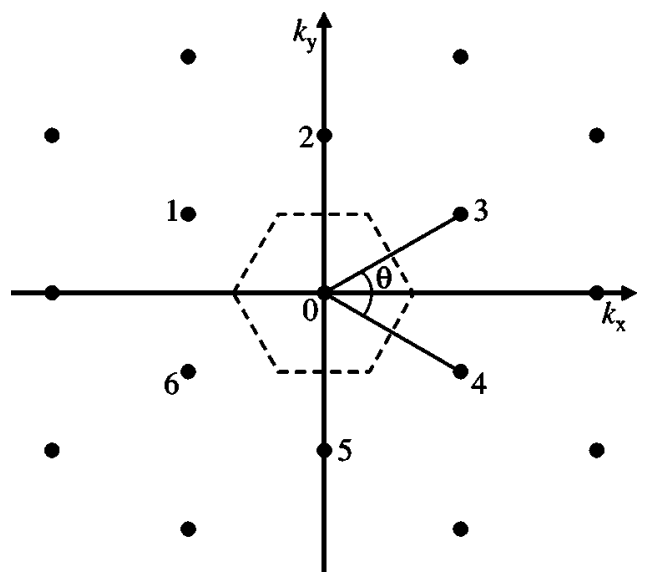

FIG. 2. First Brillouin zone (dashed hexagon) of the hexagonal lattice defined by the wave vectors of the pattern in the Fourier space.

$$
\begin{aligned}
\partial_{t} \delta a_{n}(\vec{q}, t)= & {\left[-(1+i \theta)-i\left|\vec{k}_{n}^{0}+\vec{q}\right|^{2}\right] \delta a_{n}(\vec{q}, t) } \\
& +i 2\left\{2 \sum_{l=0}^{N} \sum_{m=0}^{N} a_{l} a_{m}^{*} \delta a_{n-l+m}(\vec{q}, t)\right. \\
& \left.+\sum_{l=0}^{N} \sum_{m=0}^{N} a_{l} a_{m}\left[\delta a_{-n+l+m}(-\vec{q}, t)\right]^{*}\right\},
\end{aligned}
$$

where $\delta a_{n-l+m}(\vec{q}, t)=\delta a_{j}(\vec{q}, t)$ with $\vec{k}_{j}^{0}=\vec{k}_{n}^{0}-\vec{k}_{l}^{0}+\vec{k}_{m}^{0}$. Introducing

$\vec{\Sigma}(\vec{q}, t)=\left(\operatorname{Re}\left[\delta a_{0}(\vec{q}, t)\right], \operatorname{Im}\left[\delta a_{0}(\vec{q}, t)\right], \ldots, \operatorname{Im}\left[\delta a_{N}(\vec{q}, t)\right]\right.$

$$
\left.\operatorname{Re}\left[\delta a_{0}(-\vec{q}, t)\right], \ldots, \operatorname{Im}\left[\delta a_{N}(-\vec{q}, t)\right]\right)^{\mathrm{T}},
$$

Eq. (10) can be written as

$$
\partial_{t} \vec{\Sigma}(\vec{q}, t)=M\left(\vec{E}_{h}, \vec{q}\right) \vec{\Sigma}(\vec{q}, t)
$$

$\vec{\Sigma}(\vec{q}, t)$ includes perturbations with $+\vec{q}$ and $-\vec{q}$ since they are coupled in Eq. (10). The important point is that perturbations with different $\vec{q}$ vectors are uncoupled. Any perturbation with a vector $\vec{q}^{\prime}$ outside the first Brillouin zone of the hexagonal lattice defined by the wave vectors of the pattern $\vec{k}_{n}^{0}$ (see Fig. 2) is equivalent to another one with a vector $\vec{q}$ $=\vec{q}^{\prime}+\vec{k}_{n}^{0}$ inside.

Therefore the values of $\vec{q}$ to be considered are only those inside half of the first Brillouin zone. In this way one finds a set of $4(N+1)$ eigenvalues and eigenvectors for each vector $\vec{q}$, except for the cases $\vec{q}=\overrightarrow{0}$ and $\vec{q}=\vec{k}_{n}^{0} / 2$ where $\vec{\Sigma}(\vec{q}, t)$ has only $2(N+1)$ components. The eigenvalues may be either real or complex conjugates, and determine the linear response of the system when perturbations with $\vec{k}_{n}^{0} \pm \vec{q}$ wave vectors are applied.

As the system is translationally invariant, $E_{h}\left(\vec{x}+\vec{x}_{0}\right)$ is also a stationary solution for any fixed $\vec{x}_{0}$, the two modes

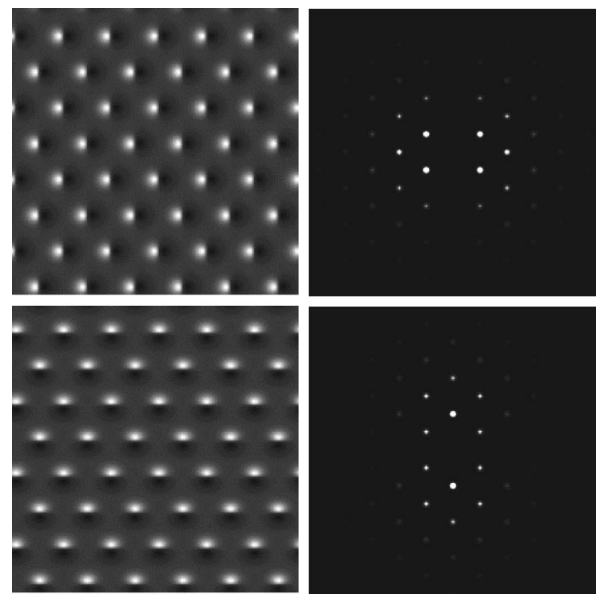

FIG. 3. Real part (left) and power spectrum (right) of the Goldstone modes $\partial_{x} E_{h}$ (top) and $\partial_{y} E_{h}$.

$\partial_{x} E_{h}$ and $\partial_{y} E_{h}$ are eigenvectors of $M\left(\vec{E}_{h}, \vec{q}=0\right)$ with zero eigenvalue. These neutrally stable modes of the linearized dynamics are the so-called Goldstone modes [1,7], and correspond to homogeneous perturbations that displace rigidly the pattern along the $x$ or $y$ direction,

$$
\partial_{x} E_{h}(\vec{x})=i \sum_{n} a_{n} k_{n x}^{0} e^{i \vec{k}_{n}^{0} \vec{x}}
$$

$$
\begin{aligned}
E_{h}(x, y)+x_{0} \partial_{x} E_{h} & =\sum_{n} a_{n}\left(1+i k_{n x}^{0} x_{0}\right) e^{i \vec{k}_{n}^{0 \vec{x}}} \\
& \simeq \sum_{n} a_{n} e^{i \vec{k}_{n}\left(x+x_{0}, y\right)}=E_{h}\left(x+x_{0}, y\right) .
\end{aligned}
$$

For the hexagonal pattern considered here, the Goldstone modes have the profile shown in Figs. 3 and 4. In the near field they have a larger amplitude at the borders of the hexagonal peaks, where the gradient is larger and show a sharp transition from negative to positive values just at the center of the peak (Fig. 4).

In a situation where the hexagonal pattern is stable, the Goldstone modes are the only neutral modes while all the other eigenvectors have eigenvalues with strictly negative real part.
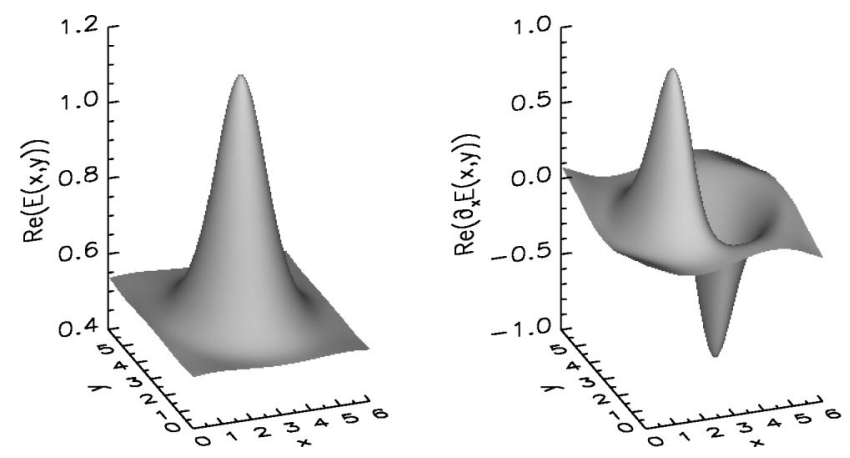

FIG. 4. Close-up of the real part of a peak of the hexagonal pattern (left) and the real part of the Goldstone mode $\partial_{x} E_{h}$ (right). 
At this point we can study the linear response to noise perturbations. Decomposing the noise term of Eq. (1) as it has been done for the field [Eqs. (8) and (9)], we obtain an extra term in the right-hand side of Eq. (11),

$$
\partial_{t} \vec{\Sigma}(\vec{q}, t)=M\left(\vec{E}_{h}, \vec{q}\right) \vec{\Sigma}(\vec{q}, t)+\vec{\Xi}(\vec{q}, t),
$$

where

$$
\vec{\Xi}(\vec{q}, t)=\left(\operatorname{Re}\left[\xi\left(\vec{k}_{0}^{0}+\vec{q}, t\right)\right], \operatorname{Im}\left[\xi\left(\vec{k}_{0}^{0}+\vec{q}, t\right)\right], \ldots,\right.
$$

$\left.\operatorname{Im}\left[\xi\left(\vec{k}_{N}^{0}+\vec{q}, t\right)\right], \operatorname{Re}\left[\xi\left(\vec{k}_{0}^{0}-\vec{q}, t\right)\right], \ldots, \operatorname{Im}\left[\xi\left(\vec{k}_{N}^{0}-\vec{q}, t\right)\right]\right)^{\mathrm{T}}$

Then, we can write an Ornstein-Uhlenbeck process [14] for the amplitude $\Theta_{i}(\vec{q}, t)$ of each eigenvector of $M\left(\vec{E}_{h}, \vec{q}\right)$,

$$
\partial_{t} \Theta_{i}(\vec{q}, t)=\lambda_{i}(\vec{q}) \Theta_{i}(\vec{q}, t)+\eta_{i}(\vec{q}, t)
$$

where $\lambda_{i}(\vec{q})$ is the $i$ th eigenvalue of $M\left(\vec{E}_{h}, \vec{q}\right)$ (ordered according to the value of its real part $\operatorname{Re}\left[\lambda_{i}(\vec{q})\right]$ $\left.\geqslant \operatorname{Re}\left[\lambda_{i+1}(\vec{q})\right]\right) . \quad \eta_{i}(\vec{q}, t)=\sum_{n=0}^{4 N+3} C_{i j}^{-1}(\vec{q}) \Xi_{j}(\vec{q}, t) \quad$ is the noise expressed in the eigenvector basis and $C(\vec{q})$ is the matrix for the change of basis as obtained diagonalizing $M\left(\vec{E}_{h}, \vec{q}\right)$. The coefficients $\delta a_{n}(\vec{q}, t)$ are related to the amplitudes of the eigenmodes $\Theta_{i}(\vec{q}, t)$ by

$$
\begin{gathered}
\operatorname{Re}\left[\delta a_{n}(\vec{q}, t)\right]=\Sigma_{i} \Theta_{i}(\vec{q}, t) C_{2 n-1 i}(\vec{q}), \\
\operatorname{Im}\left[\delta a_{n}(\vec{q}, t)\right]=\Sigma_{i} \Theta_{i}(\vec{q}, t) C_{2 n i}(\vec{q}), \\
\operatorname{Re}\left[\delta a_{n}(-\vec{q}, t)\right]=\Sigma_{i} \Theta_{i}(\vec{q}, t) C_{2 n-1+2 N i}(\vec{q}), \\
\operatorname{Im}\left[\delta a_{n}(-\vec{q}, t)\right]=\Sigma_{i} \Theta_{i}(\vec{q}, t) C_{2 n+2 N i}(\vec{q}) .
\end{gathered}
$$

The noises $\eta_{i}(\vec{q}, t)$ are Gaussian and white in time, and have cross correlations

$$
\begin{aligned}
\left\langle\eta_{i}(\vec{q}, t) \eta_{j}^{*}\left(\vec{q}^{\prime}, t^{\prime}\right)\right\rangle & =\frac{\epsilon}{2} D_{i, j}(\vec{q}) \delta\left(t-t^{\prime}\right) \delta\left(\vec{q}-\vec{q}^{\prime}\right), \\
\left\langle\eta_{i}(\vec{q}, t) \eta_{j}\left(\vec{q}^{\prime}, t^{\prime}\right)\right\rangle & =\frac{\epsilon}{2} \widetilde{D}_{i, j}(\vec{q}) \delta\left(t-t^{\prime}\right) \delta\left(\vec{q}-\vec{q}^{\prime}\right),
\end{aligned}
$$

where

$$
\begin{aligned}
D_{i, j}(\vec{q}) & =\sum_{k=0}^{4 N+3} C_{i k}^{-1}(\vec{q}) C_{j k}^{-1 *}(\vec{q}), \\
\widetilde{D}_{i, j}(\vec{q}) & =\sum_{k=0}^{4 N+3} C_{i k}^{-1}(\vec{q}) C_{j k}^{-1}(\vec{q}) .
\end{aligned}
$$

The solution of the stochastic process is then

$$
\Theta_{i}(\vec{q}, t)=e^{\lambda_{i}(\vec{q}) t} \int_{0}^{t} e^{-\lambda_{i}(\vec{q}) s} \eta_{i}(\vec{q}, s) d s .
$$

The average value of the eigenmodes amplitude is $\left\langle\Theta_{i}(\vec{q}, t)\right\rangle=0$. The correlations between the amplitudes of the eigenvectors are given by

$$
\begin{aligned}
\left\langle\Theta_{i}(\vec{q}, t) \Theta_{j}^{*}\left(\vec{q}^{\prime}, t^{\prime}\right)\right\rangle= & \frac{\epsilon D_{i j}(\vec{q})}{-4\left[\lambda_{i}(\vec{q})+\lambda_{j}^{*}\left(\vec{q}^{\prime}\right)\right]} \\
& \times\left(1-e^{\left[\lambda_{i}(\vec{q})+\lambda_{j}^{*}\left(\vec{q}^{\prime}\right)\right] t}\right) \delta\left(\vec{q}-\vec{q}^{\prime}\right),
\end{aligned}
$$

$$
\begin{aligned}
\left\langle\Theta_{i}(\vec{q}, t) \Theta_{j}\left(\vec{q}^{\prime}, t^{\prime}\right)\right\rangle= & \frac{\epsilon \widetilde{D}_{i j}(\vec{q})}{-4\left[\lambda_{i}(\vec{q})+\lambda_{j}\left(\vec{q}^{\prime}\right)\right]} \\
& \times\left(1-e^{\left(\lambda_{i}(\vec{q})+\lambda_{j}\left(\vec{q}^{\prime}\right)\right) t}\right) \delta\left(\vec{q}-\vec{q}^{\prime}\right) .
\end{aligned}
$$

In particular, the time evolution of the mean value of the squared amplitude of the eigenvectors with nonzero eigenvalue is

$$
\left\langle\left|\Theta_{i}(\vec{q}, t)\right|^{2}\right\rangle=\frac{\epsilon D_{i i}(\vec{q})}{-8 \operatorname{Re}\left[\lambda_{i}(\vec{q})\right]}\left(1-e^{2 \operatorname{Re}\left[\lambda_{i}(\vec{q})\right] t}\right)
$$

For times much longer than a characteristic time $\tau_{i}(\vec{q}) \sim$ $-1 / \operatorname{Re}\left[\lambda_{i}(\vec{q})\right]$, this mean squared amplitude reaches a stationary value

$$
\left\langle\left|\Theta_{i}(\vec{q})\right|^{2}\right\rangle=\frac{\epsilon D_{i i}(\vec{q})}{-8 \operatorname{Re}\left[\lambda_{i}(\vec{q})\right]}
$$

Equation (24) does not apply to the Goldstone modes as they have zero eigenvalue $\left[\lambda_{0}(\vec{q}=\overrightarrow{0})=0\right]$. Its time evolution is given by [from Eq. (20)]

$$
\Theta_{i}(\vec{q}, t)=\int_{0}^{t} \eta_{i}(\vec{q}, s) d s .
$$

This is a purely diffusive motion that never reaches a stationary state. Its mean squared amplitude grows linearly in time,

$$
\left\langle\left|\Theta_{0}(\vec{q}=\overrightarrow{0}, t)\right|^{2}\right\rangle=\frac{\epsilon}{8} D_{00}(\vec{q}=0) t
$$

Therefore, the linearization fails for times $t \sim 1 / \epsilon$, when the amplitude of the Goldstone modes, $\left|\Theta_{0}(\vec{q}=\overrightarrow{0})\right|^{2}$, reaches values comparable to 1 and nonlinear terms will come into play.

\section{FLUCTUATIONS IN THE NEAR FIELD}

Starting from a stationary hexagonal solution of the system equations without noise, a typical evolution of the pattern fluctuations when the noise is switched on, obtained from numerical integration of the nonlinear equations, Eq. (1) [15], is shown in Fig. 5.

From Eq. (23) we get that for short time all the modes 

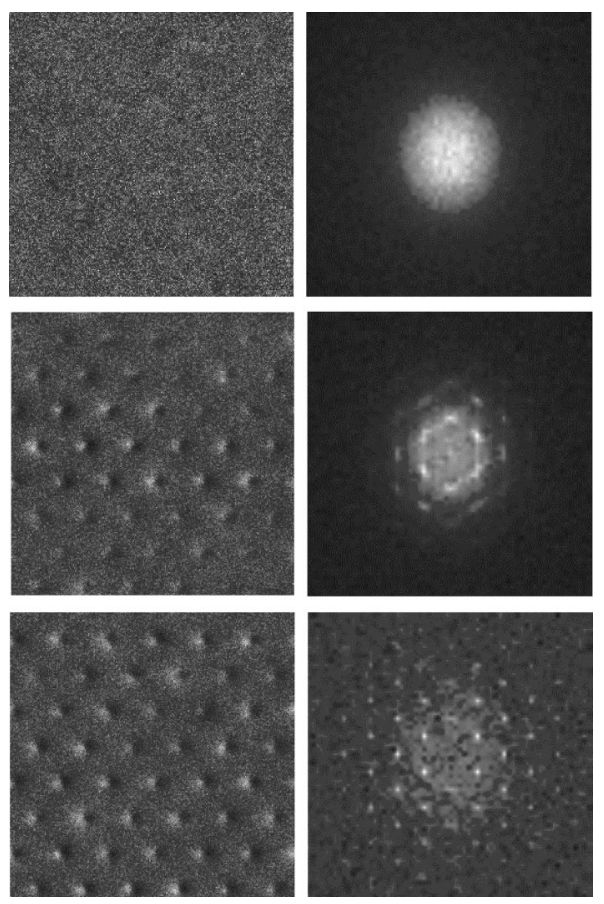

FIG. 5. Near-field (left: $\operatorname{Re}[\delta E(\vec{x})], \delta E(\vec{x})=E(\vec{x})-\langle E(\vec{x})\rangle)$ and far-field (right: $|\delta E(\vec{k})|, \delta E(\vec{k})=E(\vec{k})-\langle E(\vec{k})\rangle$ ) pattern fluctuations after switching on the noise. From top to bottom: $t=2$, $t=200$, and $t=2000$. We have considered $\epsilon=10^{-6}$.

$\Theta_{i}(\vec{q}, t)$ have a similar mean squared amplitude, which is proportional to the noise intensity and grows linearly with time,

$$
\left\langle\left|\Theta_{i}(\vec{q}, t)\right|^{2}\right\rangle=\frac{\epsilon}{4} D_{i i}(\vec{q}) t
$$

All the modes will contribute with a similar weight to the field fluctuations, and therefore there is a complete lack of structure in the field fluctuations at short times as shown in Fig. 5 (top).

As time goes on, following Eq. (23), the mean squared amplitude of the modes does not grow linearly any more. It reaches a steady state value given by Eq. (24), which is larger for the modes that have a smaller decaying rate $\operatorname{Re}\left[\lambda_{i}(\vec{q})\right]$. According to the way the eigenvalues has been ordered, for a given $\vec{q}$, the smaller decaying rate is $\operatorname{Re}\left[\lambda_{0}(\vec{q})\right]$. The eigenmodes with eigenvalue $\lambda_{0}(\vec{q})$ are the so-called soft modes $[1,7,16]$, which are connected with the Goldstone modes and for which $\lambda_{0}(\vec{q} \geqslant \overrightarrow{0}) \sim-|\vec{q}|^{2}$ (see Fig. 6).

While the Goldstone modes correspond to neutrally stable homogeneous perturbations, the soft modes correspond to weakly damped long-wavelength perturbations. For systems with a finite size $L$, the less damped of the soft modes are the ones with the smallest $\vec{q}$ vector allowed by the size of the system, $|\vec{q}|=2 \pi / L$. These modes have a decay rate propor-
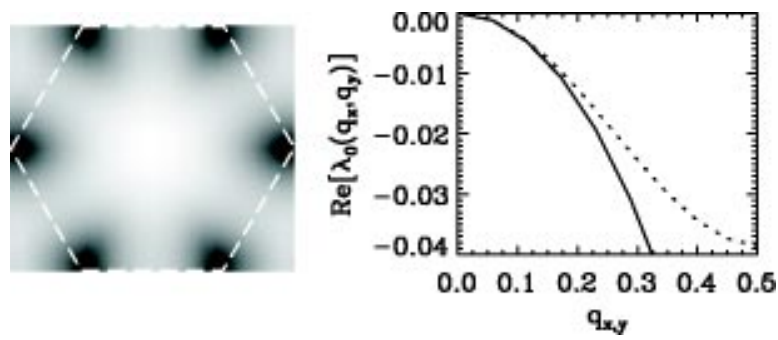

FIG. 6. Left: $\operatorname{Re}\left[\lambda_{0}(\vec{q})\right]$; darker color indicates smaller values. The white line shows the first Brillouin zone. The center of the figure corresponds to the Goldstone modes $\left(\operatorname{Re}\left[\lambda_{0}(\vec{q}=0)\right]=0\right)$. Right: transverse cut of $\operatorname{Re}\left[\lambda_{0}(\vec{q})\right]$ along the $q_{x}$ (solid line) and $q_{y}$ (dotted line) axes.

tional to $1 / L^{2}$, and therefore a stationary mean squared amplitude proportional to $\epsilon L^{2}$. The shape of one soft mode is illustrated in Fig. 7.

Its profile is similar to a Goldstone mode but with a longwavelength modulation on top of it. The wave vector of the modulation is precisely the wave vector $\vec{q}$ that identifies the soft mode. While the Goldstone modes describe an overall rigid motion of the pattern, the soft modes add some distortion, so that the pattern moves in slightly different form at different locations.

For the numerical simulations shown in Fig. 5, at $t$ $=200$ the mean squared amplitude of most of the modes has already saturated at small values, while the amplitude of the soft modes is, on average, just reaching the stationary value. Therefore, as shown in the figure (center) the field fluctuations are dominated by the soft and Goldstone modes, and short-range spatial structures start to appear. The typical correlation length of the fluctuations is determined by the wavelength of the soft modes.

According to Eq. (26) the mean square amplitude of the Goldstone mode keeps growing linearly in time and does not saturate. At times of the order $t \sim 1 / \epsilon$ the linear theory for fluctuations described in the preceding section fails (this time is around $10^{6}$ for the simulation shown in Fig. 5) Assuming the linearization is valid, at times $t \gg L^{2}$, the mean squared amplitudes of the Goldstone modes $[\sim \epsilon t$, Eq. (26)] become much larger than the amplitude of any of the soft modes and dominate the fluctuations. In our system at time $t \sim 2000$, the profile of the field fluctuations is already determined only by the Goldstone modes, as shown in Fig. 5 (bottom). In the far field, the largest fluctuations are those of the wave vectors of the pattern, while in the near field fluctuations show a long-
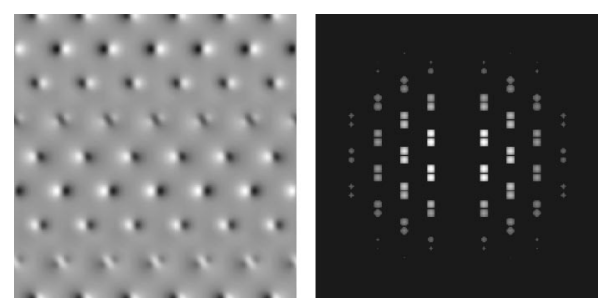

FIG. 7. Soft mode $\vec{q}=\left(0,2 \pi / L_{y}\right)$, whose decay rate is $\operatorname{Re}[\lambda(\vec{q}$ $\left.\left.=\left(0,2 \pi / L_{y}\right)\right)\right]=-0.0052$. 
range structure with strong correlation over all the system size. Integrating for longer times, the profile of the fluctuations will be dominated by macroscopic rigid displacements of the pattern. On top of that, there will be deformations generated by the soft modes.

For systems with a larger system size, the soft modes will take a longer time to reach the stationary amplitude (and, in fact, its amplitude will be larger). However, provided that $L^{2}<t \sim 1 / \epsilon$, the evolution will be basically the same, showing the three stages discussed before. If the system size is larger than $1 / \sqrt{\epsilon}$, then the stationary amplitude of the soft modes will be determined by the system nonlinearities and if $L$ is large enough, their amplitudes may have large sizes, in which case the Goldstone and soft modes will contribute to the near-field fluctuations even at quite long times $(t \sim 1 / \epsilon$.

\section{FLUCTUATIONS AND CORRELATIONS IN THE FAR FIELD}

In this section, we address on the fluctuations in Fourier space of the field, intensity, and momentum.

\section{A. Field fluctuations}

In the Fourier space the field fluctuations are also dominated by the Goldstone and soft modes. From Eqs. (12) and (13), we can see how the Goldstone modes, which have the same wave vectors than the hexagonal pattern, induce opposite and very large phase fluctuations in opposite Fourier components, which correspond to the rigid translation of the hexagons in the near field. The homogeneous component of the field is not affected by the Goldstone modes. The soft modes do not have exactly the same Fourier components than the hexagonal pattern, but they are very close, therefore their main contribution is to broaden the spots of the far field fluctuations (see the far-field for the intermediate time in Fig. 5).

\section{B. Intensity fluctuations and correlations}

The intensity fluctuations of the far-field peaks are

$$
\delta I(\vec{k})=I(\vec{k})-I_{h}(\vec{k}),
$$

where $I(\vec{k})=|E(\vec{k})|^{2}$ and $I_{h}(\vec{k})=\left|E_{h}(\vec{k})\right|^{2}$. The correlation function of the far-field intensity fluctuations of a fundamental wave vector of the pattern, for instance $\vec{k}_{3}^{0}$, with the farfield intensity fluctuations of any other wave vector $\vec{k}$ is given by

$$
C_{1}\left(\vec{k}_{3}^{0}, \vec{k}\right)=\frac{\left\langle\delta I\left(\vec{k}_{3}^{0}\right) \delta I(\vec{k})\right\rangle}{\sqrt{\left\langle\left|\delta I\left(\vec{k}_{3}^{0}\right)\right|^{2}\right\rangle\left\langle|\delta I(\vec{k})|^{2}\right\rangle}} .
$$

From the numerical integration of Eq. (1), and averaging over two hundred realizations of the noise [15], we find strong correlations between the intensity fluctuations of all the modes of the pattern, not only among the fundamental harmonics but also with the higher order ones (Fig. 8).

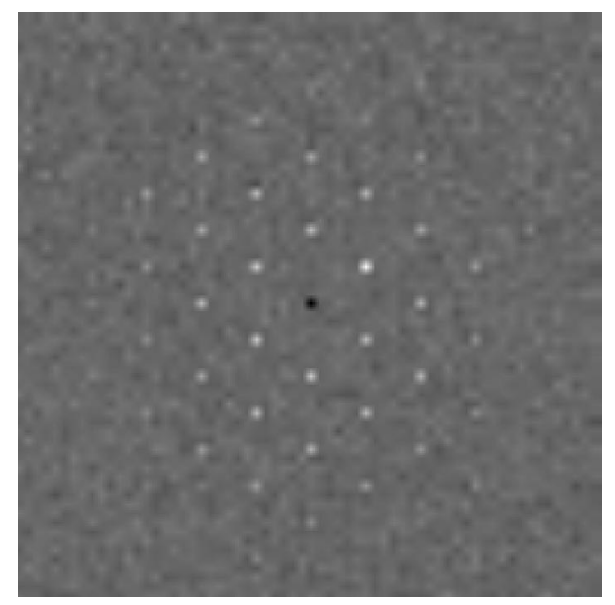

FIG. 8. Correlation function of the intensity fluctuations $C_{1}\left(\vec{k}_{3}^{0}, \vec{k}\right)$. The brightest spot takes the value 1 corresponding to the autocorrelation of $\vec{k}_{3}^{0}$. Note the strong correlation with the other six fundamental wave vectors of the pattern and with the first rings of harmonics. The correlation decays as one considers higher and higher harmonics. Note also the anticorrelation with the homogeneous component as a black spot at the center. The gray background is around 0 .

For the fundamental harmonics, the correlation $C_{1}\left(\vec{k}_{3}^{0}, \vec{k}_{i}^{0}\right)=\left\langle\delta I\left(\vec{k}_{3}^{0}\right) \delta I\left(\vec{k}_{i}^{0}\right)\right\rangle$, with $i=1,6$, is larger for the modes forming an angle $\theta=2 \pi / 3$ and smaller for $\theta=\pi / 3$ (Fig. 10). Using a six mode approximation [8,9] one finds that $N_{i}+N_{i+1}-N_{i+3}-N_{i+4}$ ( $N_{i}$ being the number of photons of the mode $i$ ) is a conserved quantity for the interaction Hamiltonian related to momentum conservation. This means that one should expect strong correlations among these sets of four Fourier modes. However, this reasoning cannot reveal which modes within these sets are more correlated in pairs. So, the stronger correlation between those modes forming a $\theta=2 \pi / 3$ angle, despite fulfilling momentum conservation, cannot be completely understood in these terms. We also find strong anticorrelations between the intensity fluctuations of the modes of the pattern and the homogeneous mode, which are related to energy conservation (Fig. 8).

Neglecting terms of order $\epsilon^{2}$, the far-field intensity fluctuations can be approximated as

$$
\delta I(\vec{k}) \approx 2 \operatorname{Re}\left[E_{h}^{*}(\vec{k}) \delta E(\vec{k})\right],
$$

where $\delta E(\vec{k})=E(\vec{k})-E_{h}(\vec{k})$. As $E_{h}(\vec{k})=\Sigma_{n} a_{n}(2 \pi)^{2} \delta\left(\vec{k}_{n}^{0}\right.$ $-\vec{k})$, we have to consider only perturbations that have the same wave vectors of the pattern $(\vec{q}=\overrightarrow{0})$. Therefore, in the linear approximation, the soft modes $(\vec{q} \geq \overrightarrow{0})$ do not contribute to the fluctuations of the far-field intensity peaks. The Goldstone modes are indeed $\vec{q}=0$ perturbations, but the intensity fluctuations associated to the Goldstone modes are $\delta I(\vec{k})=2 \operatorname{Re}\left[E_{s}(\vec{k})^{*} \partial_{x} E_{s}(\vec{k})\right]$, which [from Eqs. (6) and (12)] is exactly zero. So, in the linear approximation, neither do the Goldstone modes contribute to the fluctuations of the far-field intensity peaks. They only contribute to phase fluc- 


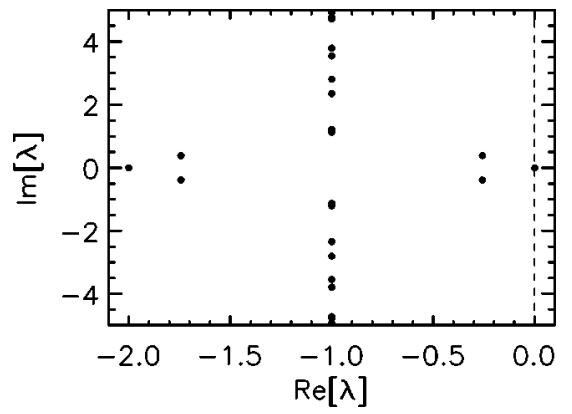

FIG. 9. Eigenvalues of the eigenvectors with $\vec{q}=\overrightarrow{0}$. There are two zero eigenvalues corresponding to the Goldstone modes. Note also the symmetry of the spectrum with respect to the axis $\operatorname{Re}[\lambda]$ $=-1$.

tuations. Therefore, fluctuations of the far-field intensity peaks have to be described by the other eigenvectors of $M\left(E_{h}, \vec{q}=\overrightarrow{0}\right)$. These eigenvectors have the same Fourier components than the hexagonal pattern, and their eigenvalues $\lambda_{i}(\vec{q}=\overrightarrow{0}),(i=1, \ldots, N)$ are shown in Fig. 9 .

Using Eqs. (16), (21), and (22), we can compute the correlation function $C_{1}\left(\vec{k}_{n}^{0}, \vec{k}_{m}^{0}\right)$ analytically,

$$
\begin{aligned}
C_{1}\left(\vec{k}_{n}^{0}, \vec{k}_{m}^{0}\right)= & 2 \operatorname{Re}\left[E_{h}^{*}\left(\vec{k}_{n}^{0}\right) E_{h}^{*}\left(\vec{k}_{m}^{0}\right)\left\langle\delta E\left(\vec{k}_{n}^{0}\right) \delta E\left(\vec{k}_{m}^{0}\right)\right\rangle\right. \\
& +E_{h}\left(\vec{k}_{n}^{0}\right) E_{h}^{*}\left(\vec{k}_{m}^{0}\right)\left\langle\delta E\left(\vec{k}_{n}^{0}\right) \delta E^{*}\left(\vec{k}_{m}^{0}\right)\right\rangle,
\end{aligned}
$$

where

$$
\begin{aligned}
\left\langle\delta E\left(\vec{k}_{n}^{0}\right) \delta E\left(\vec{k}_{m}^{0}\right)\right\rangle= & \sum_{i} \sum_{j} \frac{\epsilon}{-4\left(\lambda_{i}+\lambda_{j}\right)}\left(1-e^{\left(\lambda_{i}+\lambda_{j}\right) t}\right) \\
& \times \widetilde{D}_{i j}(\vec{q}=0)\left[C_{2 n-1 i} C_{2 m-1 j}\right. \\
& -C_{2 n i} C_{2 m j}+i\left(C_{2 m-1 i} C_{2 n j}\right. \\
& \left.\left.+C_{2 n-1 i} C_{2 m k}\right)\right]
\end{aligned}
$$

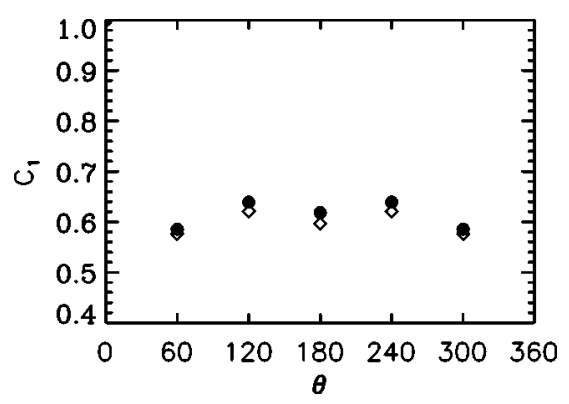

FIG. 10. Correlations between the intensity fluctuations of the fundamental wave vectors obtained from the numerical integration of Eq. (1) (rhombi) and analytically from Eq. (31) (dots).

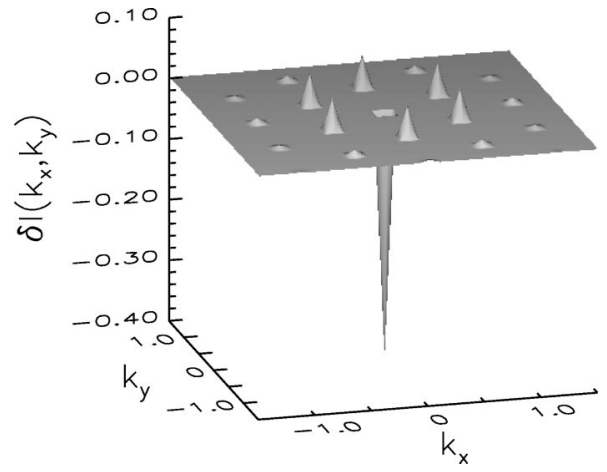

FIG. 11. Intensity fluctuations due to the eigenmodes with $\operatorname{Re}[\lambda]=-0.25$.

$$
\begin{aligned}
\left\langle\delta E\left(\vec{k}_{n}^{0}\right) \delta E^{*}\left(\vec{k}_{m}^{0}\right)\right\rangle= & \sum_{i} \sum_{j} \frac{\epsilon}{-4\left(\lambda_{i}+\lambda_{j}^{*}\right)}\left(1-e^{\left(\lambda_{i}+\lambda_{j}^{*}\right) t}\right) \\
& \times D_{i j}(\vec{q}=0)\left[C_{2 n-1 i} C_{2 m-1 j}\right. \\
& +C_{2 n i} C_{2 m j}+i\left(C_{2 m-1 i} C_{2 n j}\right. \\
& \left.\left.-C_{2 n-1 i} C_{2 m k}\right)\right]
\end{aligned}
$$

Figure 10 shows the stationary value $(t \rightarrow \infty)$ of Eq. (31) for the fundamental wave vectors of the pattern as a function of the angle $\theta$ between them. The results obtained from the linearized theory are in very good agreement with the correlations obtained from the numerical simulations of the nonlinear equation (1).

From Fig. 9 we can see that the most important eigenvectors are those associated to the complex conjugate eigenvalues with $\operatorname{Re}\left[\lambda_{2}(\vec{q}=0)\right]=\operatorname{Re}\left[\lambda_{3}(\vec{q}=0)\right]=-0.25$. This pair of eigenvectors give the strong correlation between all the Fourier components and a strong anticorrelation with the homogeneous field. The excitation of this eigenvectors implies also anticorrelations between the homogeneous mode and the off-axis Fourier components of the pattern (Fig. 11).

Some eigenvectors with $\operatorname{Re}\left[\lambda_{n}(\vec{q}=0)\right]=-1$ are finally responsible for the differences between the correlations in the fluctuations of the six first harmonics. The typical profile of one of these eigenmodes is shown in Fig. 12. The sym-

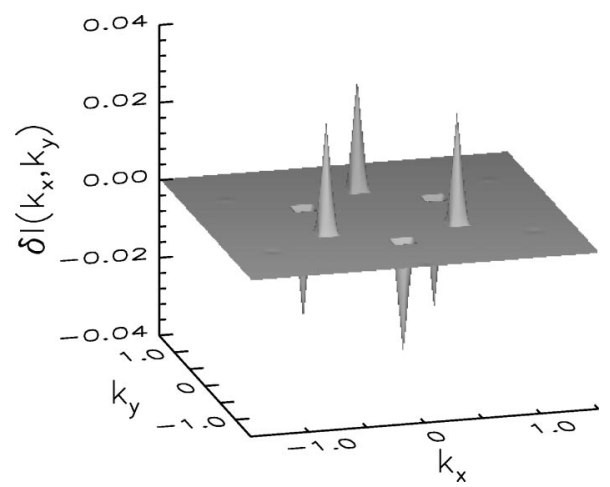

FIG. 12. Intensity fluctuations due to one of the eigenmodes with $\operatorname{Re}[\lambda]=-1$. 

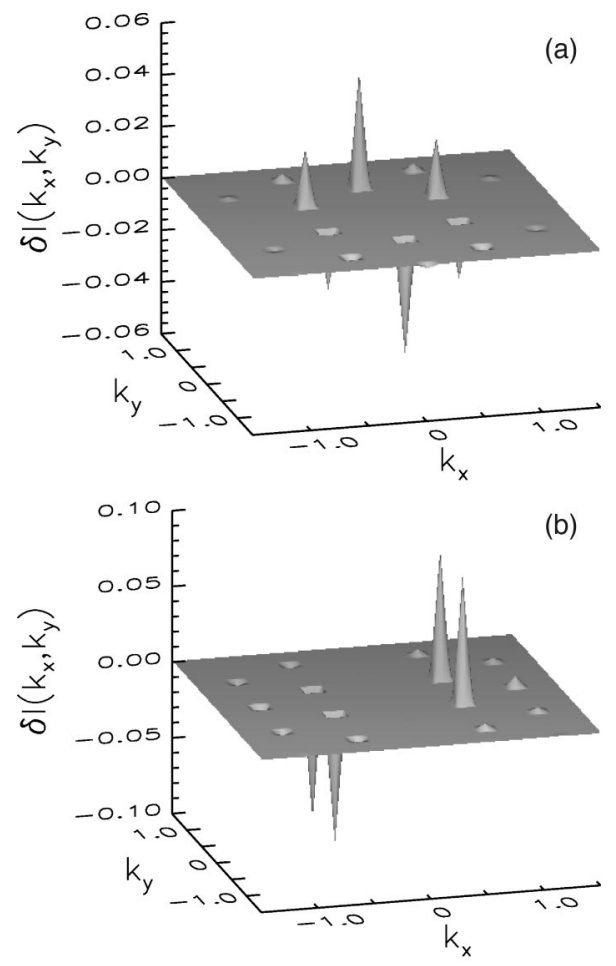

FIG. 13. Intensity fluctuations due to the two modes $\operatorname{Re}\left[\lambda_{2 N}(\vec{q}=0)\right]=\operatorname{Re}\left[\lambda_{2 N-1}(\vec{q}=0)\right]=-2$.

metry of the three peaks located at $120^{\circ}$ yields larger correlations between the fluctuations of the corresponding farfield intensity peaks.

We note that the modes that determine the correlations of the far-field intensity peaks reach stationary values in a much shorter time $\left[t \sim-1 / \operatorname{Re}\left[\lambda_{2}(\vec{q}=0)\right]=4\right]$ than the Goldstone and soft modes contributing to the near-field fluctuations $(t$ $\sim 1 / \epsilon \sim 10^{6}$, as discussed in the preceding section). As these correlations are determined by $\vec{q}=0$ perturbations, these calculations can be performed in systems with relatively small size. Increasing the system size may change the near-field profile of the fluctuations, as discussed before, but nevertheless the dynamical evolution of the modes contributing to the far-field intensity fluctuations and their mean squared stationary amplitude will be basically the same.

\section{Transverse momentum fluctuations}

We finally address the fluctuations of the transverse momentum. Without noise the total transverse momentum of the pattern is $\vec{P}=\Sigma_{n}\left|a_{n}\right|^{2} \vec{k}_{n}^{0}=0$. The noise induced fluctuations given by $\delta \vec{P}=\Sigma_{n} 2 \operatorname{Re}\left[a_{n}^{*} \delta a_{n}\right] \vec{k}_{n}^{0}$. One finds that all the eigenvectors that contribute to the far-field intensity fluctuations strictly fulfill momentum conservation except for two modes with $\lambda_{2 N}(q=0)=\lambda_{2 N-1}(q=0)=-2$, which are exactly those symmetric to the Goldstone modes with respect to the line $\operatorname{Re}[\lambda]=-1$ (Fig. 9). Therefore momentum fluctuations are determined by the two modes with maximum damping, which are shown in Fig. 13, the on the top figure breaks the conservation of the $P_{y}$ momentum component while the one in the bottom breaks the conservation of $P_{x}$.
Alternatively, it is possible to see from the classical field theory that the momentum fluctuations are damped with a coefficient -2 . A Lagrange density can be defined for Eq. (1) [17]

$$
\begin{aligned}
L= & e^{2 t}\left[a|\nabla E|^{2}+\frac{i}{2}\left(E \dot{E}^{*}-\dot{E} E^{*}\right)+i\left(E_{0} E^{*}-E_{0}^{*} E\right)\right. \\
& \left.+\theta|E|^{2}-|E|^{4}\right] .
\end{aligned}
$$

Substituting Eq. (6) in Eq. (34) and integrating over all the space we get a Lagrangian for the amplitude of the modes $a_{n}$ and for the position of the rolls in the near field $\left(\vec{x}_{0}\right)$,

$$
\begin{aligned}
L= & e^{2 t}\left[\sum_{n}\left|a_{n}\right|^{2}\left|\vec{k}_{n}\right|^{2}+\frac{i}{2} \sum_{n}\left(\dot{a}_{n}^{*} a_{n}-\dot{a}_{n} a_{n}^{*}-i 2\left|a_{n}\right|^{2} \vec{k}_{n} \dot{\vec{x}}_{0}\right)\right. \\
& +i\left(E_{0} a_{0}^{*}-E_{0}^{*} a_{0}\right)+\sum_{n}\left|a_{n}\right|^{2} \\
& \left.-\sum_{n} \sum_{n^{\prime}} \sum_{m} a_{n} a_{n^{\prime}} a_{m}^{*} a_{n+n^{\prime}+m}^{*}\right] .
\end{aligned}
$$

$\vec{x}_{0}$ is a cyclic coordinate, so its conjugate momentum,

$$
\frac{\partial L}{\partial \dot{\vec{x}}_{0}}=-i 2 e^{2 t} \sum_{n}\left|a_{n}\right|^{2} \vec{k}_{n}=-2 e^{2 t} \vec{P},
$$

is a constant. Therefore $\dot{\vec{P}}=-2 \vec{P} . \vec{P}$ is identically zero without noise. When noise is present, momentum fluctuations should satisfy

$$
\delta \dot{\vec{P}}=-2 \delta \vec{P}+\chi(t)
$$

where $\chi(t)$ is a Gaussian white noise. Therefore, momentum fluctuations have the maximum damping. The same damping coefficient for the momentum fluctuations has been found by Gatti and Mancini from a few-mode quantum formulation [9].

\section{CONCLUSIONS}

We have analyzed the fluctuations and correlations in a hexagonal pattern of a prototypical model in nonlinear optics. In the near field, fluctuations are dominated by the neutrally stable Goldstone modes associated to the translational invariance as well as by the soft modes connected with them. The soft modes destroy the long-range correlation in the fluctuations, however, in small systems these modes reach an stationary amplitude much earlier (and at a smaller value) than the Goldstone mode, so that they are important only at intermediate times. At long times the fluctuations are dominated by the Goldstone modes that correspond to rigid displacements of the overall pattern. For very large systems, both the Goldstone and soft modes may have similar amplitudes and contribute to the fluctuations.

In the far field, the most relevant effect of noise are the 
intensity fluctuations of the Fourier modes of the hexagonal pattern. At first order in noise intensity, these fluctuations are neither affected by the Goldstone modes nor by the soft modes. They are dominated by damped modes, so they reach stationary values in relatively short times. Their main characteristics are: (i) strong correlations between the intensity fluctuations of any arbitrary pair of the six fundamental wave vectors of the pattern, and also with their higher harmonics, (ii) larger correlation between intensity fluctuations of the Fourier modes forming $120^{\circ}$ angles than between modes forming $180^{\circ}$, and (iii) strong anticorrelations between the zero wave vector and the pattern Fourier modes. Finally, only the eigenmodes with maximum damping contribute to the fluctuations of the total transverse momentum, therefore the total transverse momentum has the least possible fluctuations.

Our results are obtained from both semianalytical calculations based on linearization around the hexagonal pattern and from numerical simulations of the nonlinear system. Some of our results and predictions are very general, and depend only on basic symmetry properties of the system, such as the translational invariance. Thus we expect that similar structure and properties of the fluctuations and correlations can be found in other nonlinear systems displaying hexagonal patterns.

From a computational point of view, correlations in the far-field intensity peaks are practically independent of the system size, and therefore can be calculated accurately in relatively small systems, provided all the relevant harmonics of the pattern are considered. Good statistics can be obtained on integrating the nonlinear equations over relatively short times (even though the near-field fluctuations are quite far away from reaching a stationary value). Of course, alternatively a linear semianalytical approach as the one described in Sec. III can also be used to calculate far-field correlations.

\section{ACKNOWLEDGMENTS}

The authors acknowledge helpful discussions with M. San Miguel and R. Zambrini, and financial support from the EC TMR Network QSTRUCT (FMRXCT960077) and from the MCyT, Spain (Project Nos. PB97-0141-C02-02, BFM20001108, and BFM2001-0341-C02-02).
[1] D. Foster, Hydrodynamic Fluctuations, Broken Symmetry and Correlation Fluctuations (Addison-Wesley, Redwood City, CA, 1983).

[2] J. Viñals, E. Hernández-García, M. San Miguel, and R. Toral, Phys. Rev. A 44, 1123 (1991); E. Hernández-García, J. Vĩnals, R. Toral, and M. San Miguel, Phys. Rev. Lett. 70, 3576 (1993); M. San Miguel, in Growth Patterns in Physical Sciences and Bilogy, edited by J. M. García-Ruíz et al. (Plenum Press, New York, 1993).

[3] L.A. Lugiato and F. Castelli, Phys. Rev. Lett. 68, 3284 (1992).

[4] L.A. Lugiato and G. Grynberg, Europhys. Lett. 29, 675 (1995); F. Castelli and L.A. Lugiato, J. Mod. Opt. 44, 765 (1997).

[5] M. Hoyuelos, Pere Colet, and M. San Miguel, Phys. Rev. E 58, 74 (1997).

[6] M. Hoyuelos, Alice Sinatra, Pere Colet, Luigi Lugiato, and M. San Miguel, Phys. Rev. A 59, 1622 (1998).

[7] R. Zambrini et al., Phys. Rev. A 62, 063801 (2000).

[8] G. Grynberg and L.A. Lugiato, Opt. Commun. 101, 69 (1993).

[9] A. Gatti and S. Mancini, Phys. Rev. A 65, 013816 (2001).

[10] L.A. Lugiato and R. Lefever, Phys. Rev. Lett. 58, 2209 (1987); W.J. Firth, A.J. Scroggie, G.S. McDonald, and L.A. Lugiato, Phys. Rev. A 46, R3609 (1992).
[11] M. Aguado, R.F. Rodriguez, and M. San Miguel, Phys. Rev. A 39, 5686 (1989).

[12] D. Gomila and P. Colet (unpublished).

[13] P. Coullet and G. Iooss, Phys. Rev. Lett. 64, 866 (1990).

[14] C.W. Gardiner, Handbook of Stochastic Methods (SpringerVerlag, Berlin, 1983).

[15] We integrate Eq. (1) using a pseudospectral method where the linear terms in Fourier space are integrated exactly while the nonlinear ones are integrated using a first-order in time approximation, following the scheme described by M. San Miguel and R. Toral, in Instabilities and Nonlinear Equilibrium Structures, edited by E. Tirapegui, J. Martinez, and R. Tiemann (Kluwer, Dordrecht, 2000). The random number generator used is described in R. Toral and A. Chakrabarti, Comput. Phys. Commun. 74, 327 (1993), A rectangular lattice of size $256 \times 256$ points was used. In Fourier space we took $\Delta k_{y}=1 / 8$ and $\Delta k_{x}=\sin \left(60^{\circ}\right) / 6$, which corresponds in real space to a system of size $L_{y} \approx 50.3$ and $L_{x} \approx 43.5$. The time step for integration was $d t=0.001$.

[16] S.K. Ma, Statistical Mechanics (World Scientific, Singapore, 1985).

[17] W.J. Firth and A. Lord, J. Mod. Opt. 43, 1071 (1996). 